\title{
ACCESS: Design and Sub-system Performance
}

\author{
Mary Elizabeth Kaiser ${ }^{a}$, Matthew J. Morris ${ }^{a}$, Stephan R. McCandliss ${ }^{a}$, Bernard J. Rauscher ${ }^{b}$, \\ Randy A. Kimble ${ }^{b}$, Jeffrey W. Kruk ${ }^{b}$, Russell Pelton ${ }^{a}$, D. Brent Mott ${ }^{b}$, Yiting Wen ${ }^{b}$, Roger \\ Foltz $^{b}$, Manuel A. Quijada ${ }^{b}$, Jeffery S. Gum ${ }^{b}$, Jonathan P. Gardner ${ }^{b}$, Duncan M. Kahle ${ }^{b}$, \\ Dominic J. Benford ${ }^{b}$, Bruce E. Woodgate ${ }^{b}$, Edward L. Wright ${ }^{c}$, Paul D. Feldman ${ }^{a}$, Murdock \\ Hart $^{a}$, H. Warren. Moos ${ }^{a}$, Adam G. Riess ${ }^{a d}$, Ralph Bohlin ${ }^{d}$, Susana E. Deustua ${ }^{d}$, W. V. \\ Dixon $^{d}$, David J. Sahnow ${ }^{d}$, Robert Kurucz ${ }^{e}$, Michael Lampton ${ }^{f}$, and Saul Perlmutter ${ }^{g}$ \\ a Johns Hopkins University, 3400 North Charles Street, Baltimore, MD, USA 21218; \\ ${ }^{b}$ NASA Goddard Space Flight Center, Greenbelt, MD USA 20771; \\ ${ }^{c}$ University of California Los Angeles, Los Angeles, Los Angeles, CA 90024; \\ ${ }^{d}$ Space Telescope Science Institute, San Martin Drive, Baltimore, MD, USA 21218; \\ ${ }^{e}$ Harvard Smithsonian Center for Astrophysics, Garden Street, Cambridge, MD, USA 02139;

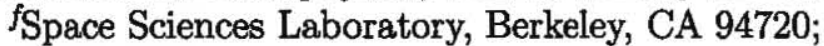 \\ ${ }^{g}$ University of California, Berkeley, Berkeley, CA 94720
}

\begin{abstract}
Establishing improved spectrophotometric standards is important for a broad range of missions and is relevant to many astrophysical problems. ACCESS, "Absolute Color Calibration Experiment for Standard Stars", is a series of rocket-borne sub-orbital missions and ground-based experiments designed to enable improvements in the precision of the astrophysical flux scale through the transfer of absolute laboratory detector standards from the National Institute of Standards and Technology (NIST) to a network of stellar standards with a calibration accuracy of $1 \%$ and a spectral resolving power of 500 across the $0.35-1.7 \mu \mathrm{m}$ bandpass.
\end{abstract}

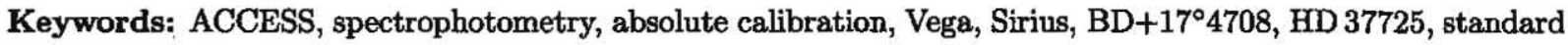
stars, NIST

\section{INTRODUCTION}

As a probe of the expansion history of the universe, using SNeIa to distinguish dark energy models from one another levies a requirement for $1 \%$ precision in the cross-color calibration of the $\mathrm{SNe} \mathrm{Ia}$ flux across a bandpass extending from $0.35-1.7 \mu \mathrm{m}$. However, the systematic errors in the flux calibration network spanning the visible through the NIR currently exceed $1 \%$. The astrophysical flux scale is ultimately pinned to a single star, Vega $a^{1,2}$ through ground based measurements of a fundamental standard, a melting point black body furnace. ${ }^{3}$ Since that fundamental calibration in 1970, there have been significant technological advances in detectors, telescopes and instruments, and the precision of the fundamental laboratory standards used to calibrate these instruments. These technologies have not yet been transferred to the fundamental astrophysical flux scale across the visible to NIR bandpass. In addition, Vega, the ultimate fundamental stellar standard for the astrophysical flux scale, has proven to be a poor choice for the following reasons. Vega is a pole-on rotator. ${ }^{4-6}$ As a result, it presents a range of effective temperatures and is difficult to model uniquely. It exhibits an infrared excess that arises from its circumstellar debris disk. And, it is too bright to be observed with today's premier optical telescopes.

ACCESS - "Absolute Color Calibration Experiment for Standard Stars", is a series of rocket-borne sub-orbital missions and ground-based experiments that will enable measurement of the absolute flux for a limited set of primary standard stars to be established using calibrated detectors as the fundamental metrology reference. ${ }^{7,8}$ These experiments are designed to obtain an absolute spectrophotometric calibration accuracy of $<1 \%$ in the $0.35-1.7 \mu \mathrm{m}$ bandpass at a spectral resolution greater than 500 by directly tracing the observed stellar fluxes to NIST irradiance standards. Transfer of the NIST detector standards to our target stars (Table 1) will produce an absolute calibration of these standards in physical units, including the historic absolute standard Vega, the Sloan Digital Sky Survey (SDSS) standard BD+17 $4708,{ }^{9}$ and the Spitzer IRAC standard HD 37725. ${ }^{10}$

Send correspondence to M. E. Kaiser: E-mail: kaiser@pha.jhu.edu 


\section{OBSERVING STRATEGY}

ACCESS has adopted a multi-faceted strategy for reducing systematic errors and establishing the first members in a network of standard stars calibrated to $1 \%$ precision.

First in this multi-pronged approach is the elimination of the Earth's atmosphere as a spectral contaminant. One of the prime impediments to obtaining a precise flux calibration in the NIR is the plethora of hydroxyl (OH) lines that are formed high $(\sim 70 \mathrm{~km}-\sim 89 \mathrm{~km})$ in the Earth's atmosphere. ${ }^{11}$ These spectral lines are strong, numerous - extending across $0.85 \mu \mathrm{m}<\lambda<2.25 \mu \mathrm{m}$, and highly variable on short timescales. Correcting ground based observations to $1 \%$ precision for absorption by the Earth's atmosphere in the NIR is prohibitive. Spectral contamination by $\mathrm{OH}$ emission remains daunting even at balloon altitudes.

As a result, the ACCESS observations will execute from a sounding rocket platform completely above the Earth's atmosphere. The typical launch trajectory for a Black Brant IX sounding rocket carrying a $1000 \mathrm{lb}$ payload yields an apogee of $300 \mathrm{~km}$ with the time above $100 \mathrm{~km}$ in excess of 400 seconds. Observing at an altitude exceeding $100 \mathrm{~km}$ eliminates the challenging problem of measuring the residual atmospheric absorption and strong atmospheric emission seen by ground-based observations and even by observations conducted at balloon altitudes. The number, strength, and variability of the $\mathrm{OH}$ lines was an important factor in the selection of a sounding rocket platform for the ACCESS observations.

Potential ACCESS stellar flux standards were selected based upon their ability to serve as precise calibration standards in both the visible and the NIR spectral region. This required that they (1) have existing photometric heritage, (2) present a stellar atmosphere capable of being precisely modelled, and are (3) bright enough to obtain a spectrum with $\mathrm{S} / \mathrm{N}$ of $\mathbf{2 0 0}$ in a single rocket flight. Since our program includes observations of Sirius and Vega, which are $12 \mathrm{hr}$ apart on the sky, this also imposed the requirement that (4) additional targets be within $45^{\circ}$ of the zenith on either the Sirius or Vega flight. To optimally utilize the flight time and to eliminate a step in the calibration transfer to larger telescopes, we required that (5) all targets other than Vega and Sirius be fainter than 6th magitude. The current set of ACCESS targets is presented in Table 1.

Further reduction of systematic errors will be achieved using a spectrograph with sensitivity extending from the visible through the NIR to eliminate this cross-calibration. Key to achieving this broad bandpass is the use of WFC3 heritage $\mathrm{HgCdTe}$ detector whose sensitivity extends from below $4000 \AA$ to $\sim 1.7 \mu \mathrm{m}$.

Another component of the calibration strategy is to establish an a priori error budget and track it.

Multiple methods will be used to determine the payload sensitivity through NIST traceable sub-system and end-to-end payload calibrations. A fundamental component of the calibration strategy is the use of NIST calibrated photodiode detector (irradiance) standards to establish NIST traceability. The NIST traceable photodiode irradiance standards will be used to establish the artificial star used to calibrate the integrated telescope and spectrograph. In addition to both an absolute and relative calibration of the payload, and hence the stars, the irradiance standards will establish the calibration system in fundamental physical units. End-to-end payload calibrations will be performed with both NIST continuum and emission line radiance standards as cross-checks for systematic errors.

ACCESS includes an On-board Calibration Monitor (OCM) to track the payload performance in the field prior to launch and while parachuting to the ground post-observation. The OCM will be calibrated at the same time as the payload and re-calibrated and monitored between flights.

A S/N of 200 per spectral resolution element over the full spectral bandpass will be achieved for the brighter targets in the sample. $0^{\text {th }} \mathrm{V}$ magnitude stars Sirius and Vega will require subsecond integration times using subarray readouts to avoid saturation of the detector. For the fainter targets (e.g., BD $+17^{\circ} 4708$ ) a 400 second observation yields a $\mathrm{S} / \mathrm{N}$ of 200 per spectral resolution element down to the Balmer edge. Additional binning can further increase the background subtracted signal-to-noise ratio of the acquired spectrum.

\section{ACCESS TELESCOPE AND SPECTROGRAPH}

The ACCESS telescope is a Dall-Kirkham cassegrain with a 15.6-inch diameter Zerodur primary mirror. The telescope mirrors have been coated with aluminum and a protective $\mathrm{MgF}_{2}$ overcoat (section 5). This coating 


\begin{tabular}{lllllll}
\hline Target & Alt Name & R.A. (2000) & Dec (2000) & Sp. Type & Vmag & Hmag \\
\hline & & & & & & \\
HD 37725 & & 054154 & +291751 & A3V & 8.35 & 7.92 \\
HD 48915 & Sirius & 064509 & -164258 & A1V & -1.47 & -1.39 \\
HD 84937 & & 094856 & +134446 & sdF5 & 8.28 & 7.12 \\
HD 165459 & & 180231 & +583738 & A1V & 6.86 & 6.63 \\
HD 172167 & Vega & 183656 & +384701 & A0V & +0.03 & -0.03 \\
BD+17 4708 & & 221131 & +18533 & sdF8 & 9.47 & 8.11 \\
\hline
\end{tabular}

Table 1. ACCESS primary and secondary targets.
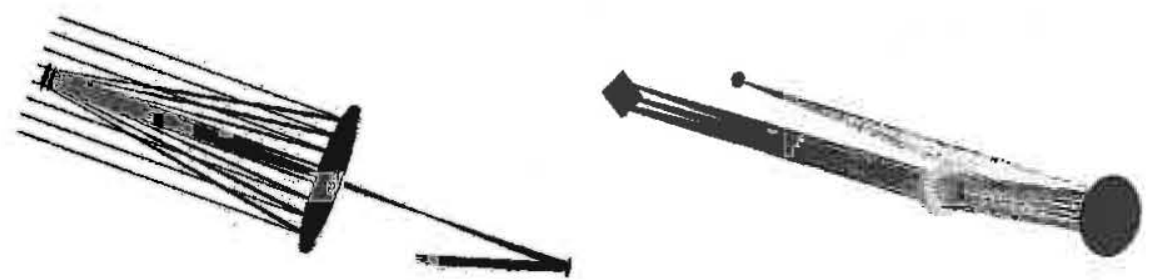

Figure 1. Left: Ray trace view of ACCESS. Parallel rays from the star enter the Dall-Kirkham Cassegrain telescope at left and are incident on the primary mirror (center of figure). The telescope secondary is at left in the figure and the grating is the optical surface at the extreme right. Right: Ray trace view of the ACCESS spectrograph illustrating the grating on the right, the cross-dispersing prism, and the first three orders dispersed by the grating and incident on the detector at left.

was chosen in order to retain sensitivity in the $3500-4000 \AA$ range where the higher order Balmer lines provide leverage for stellar atmosphere modeling. The telescope feeds a low-order echelle spectrograph.

The spectrograph is a Rowland circle design configured as an echelle (Fig. 1) used in 1st (9000-19000 $\AA$ ), 2nd $(4500-9500 \AA)$ and 3rd orders $(3000-6333 \AA)$, with some sensitivity in the 4th order. It consists of just two figured optical elements: a concave diffraction grating with a low ruling density, and a Féry prism ${ }^{12}$ with spherically figured surfaces placed in the converging beam. The prism provides astigmatism correction and cross-disperses the spectrum.

The flight grating is a mechanically ruled (blazed), quad-partite, concave grating with a radius of curvature of $400.7 \mathrm{~mm}$, a groove density of 45 lines per $\mathrm{mm}$, and a groove depth of $\sim 660 \mathrm{~nm}$. The spectrograph design is optimized to use the grating through a range of small diffraction angles. This restricts the astigmatism generated by the Rowland circle mount configuration.

The prism is fabricated from LLF1 glass to provide high, relatively uniform, transmission and sufficient dispersion to separate orders and isolate the wings of the line spread function of adjacent orders on the detector across the bandpass extending from $3500 \AA$ to $1.8 \mu \mathrm{m}$ (Fig. 2).

The resolution of the spectrograph depends on the telescope point spread function (PSF) and the size of the detector pixels. For the telescope PSF of $1.17^{\prime \prime}$ (as achieved on recent flights with a similar design) the $18 \mu \mathrm{m}$ pixels of the detector provide critical sampling and produce constant wavelength resolution elements in each order, giving a resolving power ranging between 500 and 1000 (Fig. 2).

The spectrograph optical elements are sealed in a vacuum housing mounted to the back of the primary mirror base plate. An angled mirrored plate with a $1 \mathrm{~mm}$ aperture in the center located at the telescope focus serves as the slit jaw, ${ }^{13}$ allowing light to enter the spectrograph while reflecting the region surrounding the target into an image-intensified video camera for real-time viewing and control by the operator on the ground. An entrance window sits behind the slit jaw and provides the seal for the spectrograph vacuum housing. The grating and prism cross disperser are mounted inside along with a set of baffles. The detector housing is connected to the spectrograph housing via a bellows that is used as part of the focus adjustment system. Fine threaded rods welded into the spectrograph housing and passing through the detector housing flange provide the focus adjustment mechanism. 


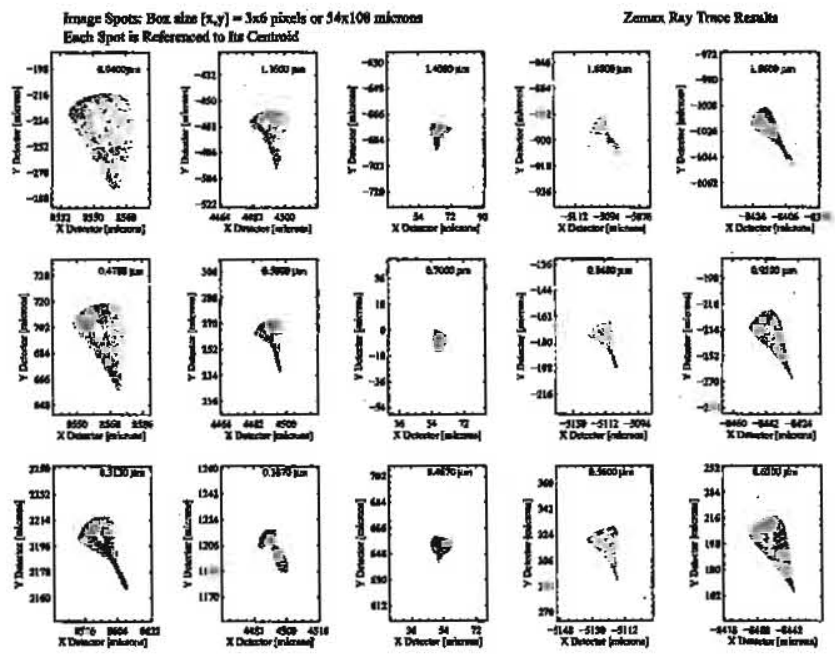

Figure 2. Spot diagrams for the optical layout at a selection of wavelengths spanning the ACCESS bandpass. Each box is labelled in microns and each spot is referenced to the chief ray. In general, the ray trace yields images that are within 2 pixels in the dispersion direction, $\sim 4$ pixels in the cross-dispersion (vertical) direction.
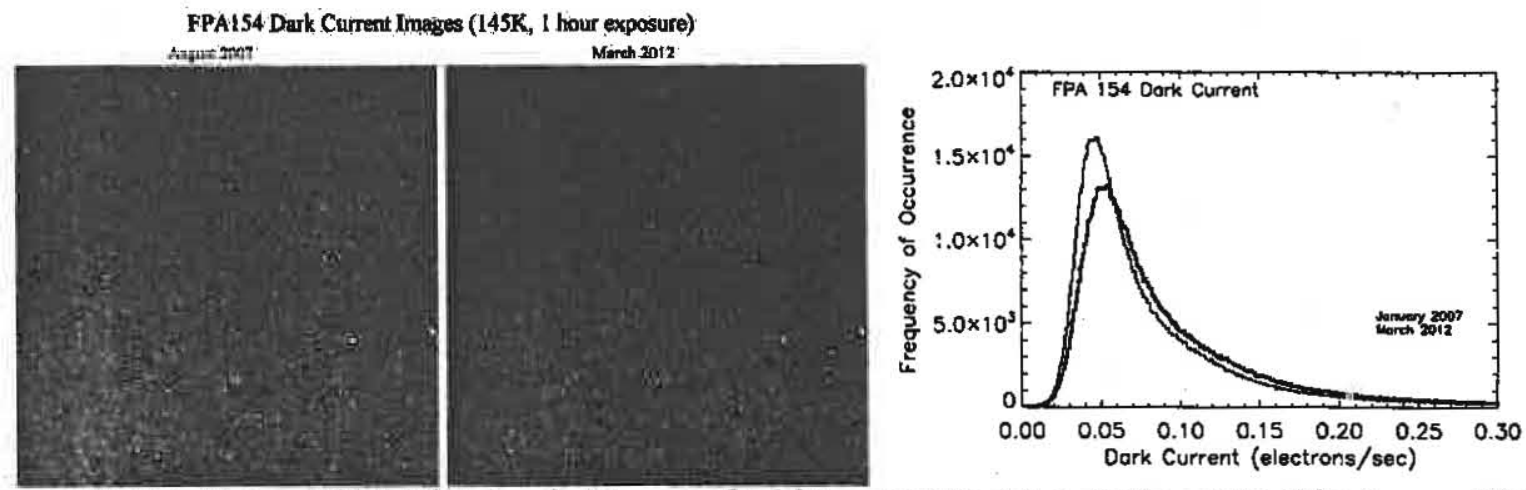

Figure 3. Left \& Center: FPA154 detector dari images of a 1 hour exposure at two epochs separated by 5 years. Right: Histogram of the dark current at the two epochs. The dark current is $\sim 0.05$ electrons/sec for the pixel population and has not varied significantly. Data at each epoch were taken with the detector temperature at $145 \mathrm{~K}$.

\section{DETECTOR PERFORMANCE}

The focal plane detector array is a HST/WFC3 heritage HgCdTe device with a $1024 \times 1024$ pixel format and $18 \mu \mathrm{m} \times 18 \mu \mathrm{m}$ pixels. The detector composition is tailored to produce a long-wavelength cutoff at $\sim 1.7 \mu \mathrm{m}$. The CdZnTe growth substrate is removed to provide high near-IR quantum efficiency (QE) and response through the visible to $3500 \AA$. The flight detector QE (Fig. 4) Is $~ 30 \%$ at $5000 \AA$. The band gap corresponding to the $1.7 \mu \mathrm{m}$ cutoff yields low dark current at operating temperatures near $140 \mathrm{~K}$ (Fig. 3) and results in a detector that is relatively insensitive to thermal background radiation. The detector assembly consists of a two part molybdenum interface to match the CTE of the molybdenum base plate mounted on the detector as supplied by Teledyne. One molybdenum block is permanently stycast to the detector molybdenum, the second molybdenum block bolts to the first and contains the thermal feedback and monitoring system. Titanium flexures mounted between the molybdenum and G10-cryogenic standoffs accommodate the CTE difference between these materials. The G10-CR standoffs provide thermal isolation between the $140 \mathrm{~K}$ detector operating temperature and the detector housing (Fig. 7). A detector radiation shield, baffle, and cold stop will be cooled to $\sim 200 \mathrm{~K}$ to reduce the thermal background to an acceptable level. Cooling of the baffles is achieved through thermal inertia of a cold block that has been cooled with a $\mathrm{LN}_{2}$ pass through prior to launch. A Stirling cooler provides cooling for the detector. 

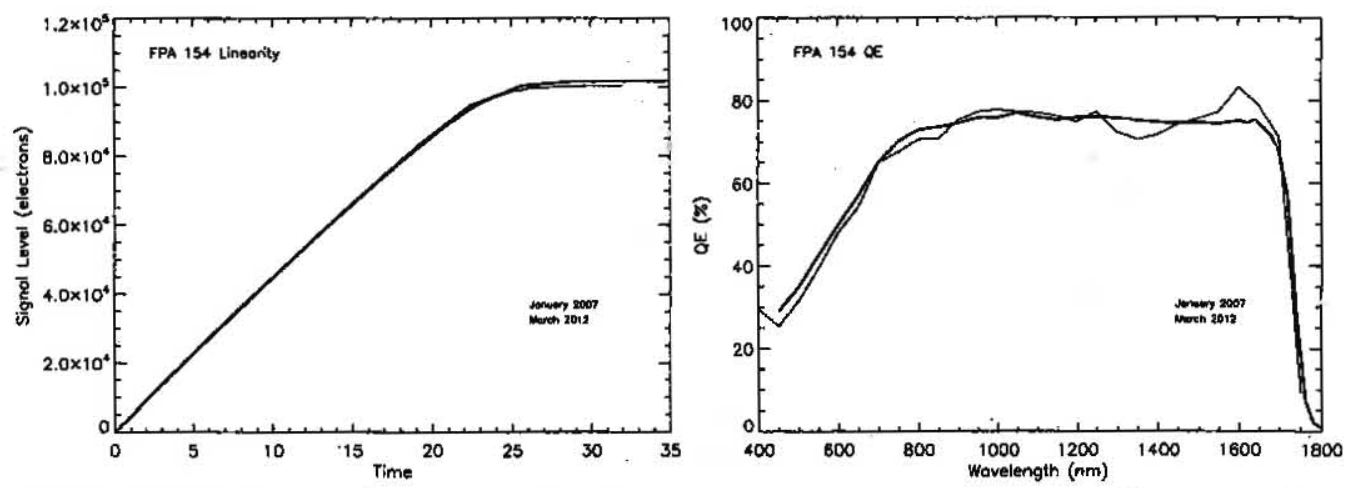

Figure 4. Left: FPA154 detector linearity and full well capacity taken at two epochs separated by 5 years. Right: Quantum efficiency of FPA154. The QE data has been corrected for interpixel capacitance. Data at each epoch were taken with the detector temperature at $145 \mathrm{~K}$. These metrics have not varied within measurement error across the two epochs.

The ACCESS detectors were selected based upon a suite of initial characterization tests conducted by GSFC in 2007 and the re-testing in 2011-2012 of key performance metrics such as dark current, read noise and quantum efficiency and to extend the QE measurements to shorter $(4000 \AA)$ wavelengths. Reciprocity failure testing has been conducted to measure the count rate dependent non-linearity. ${ }^{14}$

FPA 154 and FPA 166 showed no significant performance changes over a $\sim 5$ year baseline (2007 to 2012) between measurements. Figure 4 illustrates the stability of the full well-linearity and quantum efficiency performance metrics for FPA 154. Illuminated flat field images at $1200 \mathrm{~nm}$ with different exposure times were acquired at each epoch. The images at each epoch were differenced and ratioed (Figure 5). For the ratio image, the data from 2012 was scaled by the ratio of the quantum efficiences (1.0101) at $1200 \mathrm{~nm}$ between 2007 and 2012 . No anomalies are evident in either the ratio image or the histogram.

Sounding rocket vibration test loads for the Black Brandt IX vehicle are a random vibration spectrum at $12.77 \mathrm{~g}$ rms and a sine sweep from $5-2000 \mathrm{~Hz}$ with discrete load levels of $1.5 \mathrm{~g}(24-110 \mathrm{~Hz}), 3.5 \mathrm{~g}(110-800 \mathrm{~Hz})$, and $10 \mathrm{~g}(800-2000 \mathrm{~Hz})$. Concerns regarding survivability under launch loads of the detector assembly interface to the external circuitry used to drive the signal from the detector to the controller resulted in a redesign of the detector's external buffer amplifier circuitry. These FPA electronics were reconfigured from a PCB to a compact and lightweight flex circuit design Figure 7 to enable launch load survival. This compact footprint also enabled packaging of the detector assembly. For a Rowland circle optical design the telescope focus and the spectrograph focus lie on a circle of a given radius. As a result, the cross-dispersed beam at the spectrograph focal plane and the incoming beam at the telescope focus are in close proximity given the constraining 17.26-inch OD for the rocket skin.

\section{MIRROR COATINGS}

The primary and secondary telescope mirrors and the auto-collimating 18-inch flat mirror have been coated with aluminum and a $\mathrm{MgF}_{2}$ protective overcoat layer: Prior to coating the flight mirrors, witness samples were mounted along the radius, coated, and measured to determine the azimuthal and expected radial dependence of the reflectance (Figure 8). As expected from modeling of earlier coating tests, a slight radial dependence in the reflectance was found and modeled as a slight $(50 \AA)$ radial variation in the thickness of the $\mathrm{MgF}_{2}$ overcoat layer. The reflectance performance of the coating rest runs and the final coating run for these mirror is presented in Figure 8 and Table 2. The coatings are thickest at the center of the mirror and decrease in thinkness by $\sim 5 \AA$ per inch. The dashed curves measure the reflectance of test data at different radii spanning the extent of the mirror. From inspection of the dashed curves, the higher reflectance resulting from the thinner layer of $\mathrm{MgF}_{2}$ at increasing radii is apparent.

The reflectance performance meets the ACCESS requirements. Spectrally, the radial spatial dependence is largest in the blue region of the ACCESS bandpass, with a maximum variation of $\sim 0.4 \%$ occurring at $3500 \AA$ to 

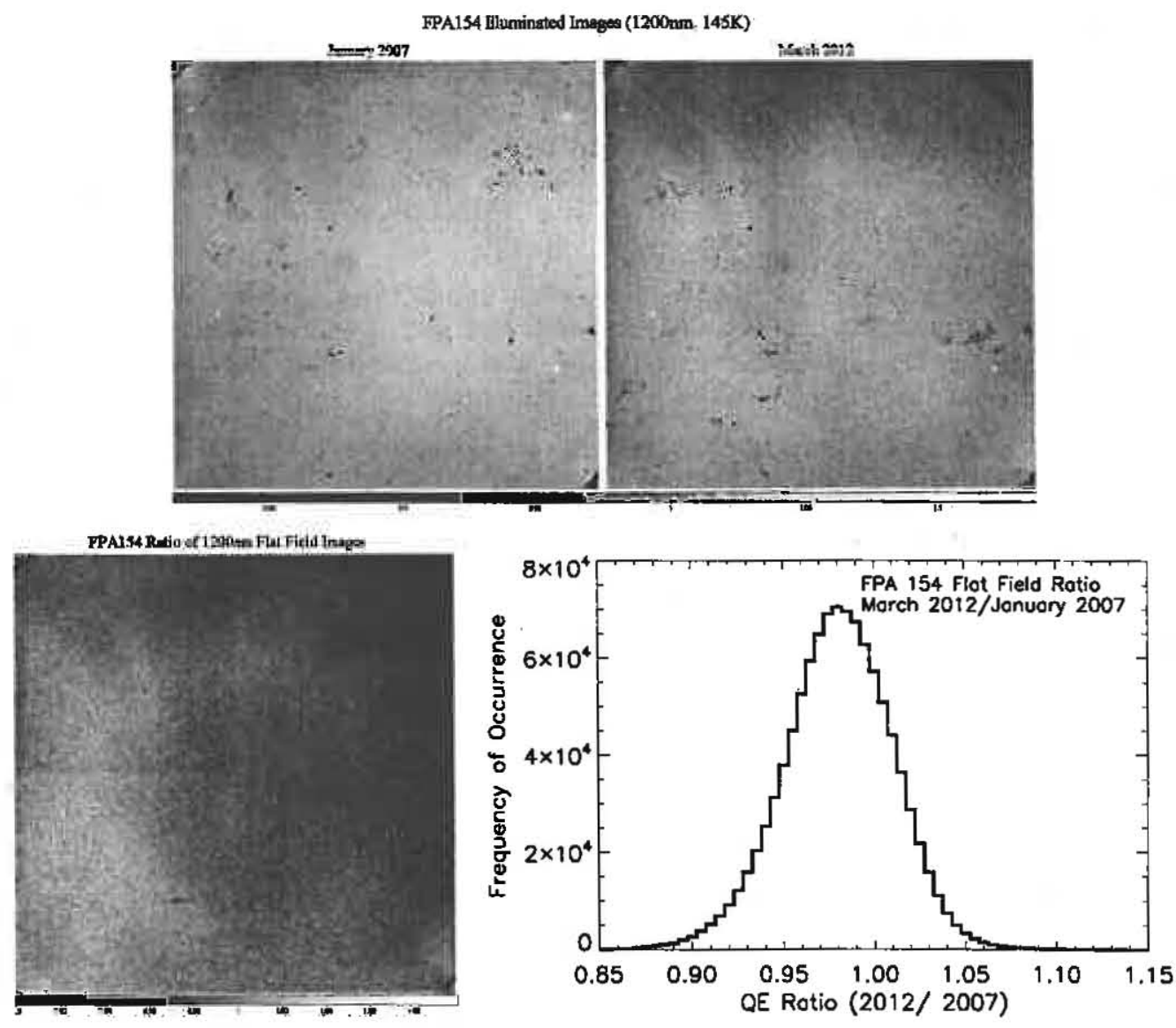

Figure 5. Top: Flat field difference images taken at $1200 \mathrm{~nm}$ as part of the test suite for assessing the detector qe, linearity, and full well performance. Lighter pixels have higher response. Bottom Left: Ratio image of the flat fields taken at the 2007 and 2012 epochs. The flat field image from 2012 was divided by the image from 2007, thus darker pixels have decreased response. Bottom Right: Histogram of the flat field ratio. 


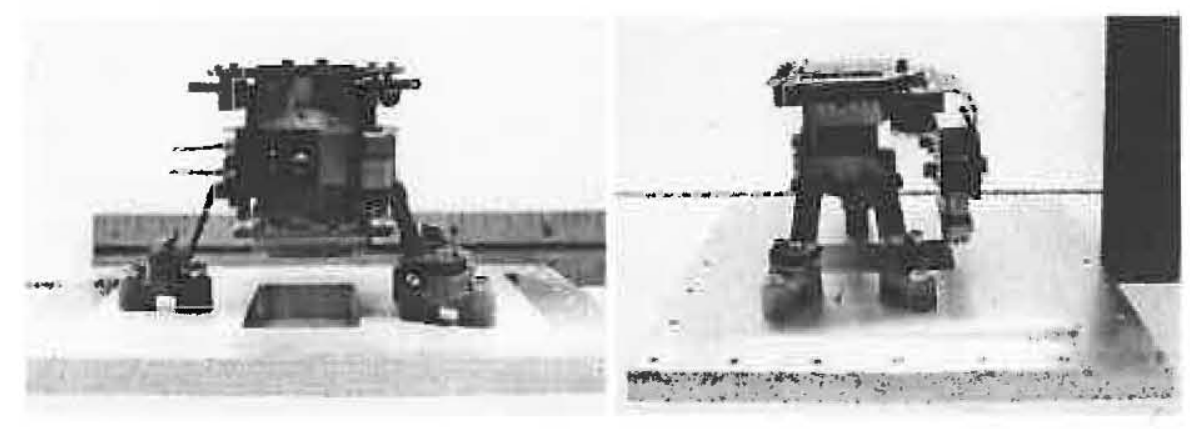

Figure 6. Left: Front view of detector with fight mount prior to vibration testing with launch loads. The (black) heater element of the thermal control system is mounted on a molybdenum block. Not mounted at the time of this picture are the thermal control diode and the thermal monitor diode. These two thermal diodes are located on the molybdenum face opposite the heater and on the molybdenum arm just above the two titanium flexures, respectively. The titanium flexures are mounted on G10-cryogenic standoffs that provide themal isolation between the $140 \mathrm{~K}$ detector temperature and the detector housing. The detector cold finger attaches to the copper block below the heater. Right: Side view of detector with flight mount. Note: the bolts were staked prior to vibration testing.

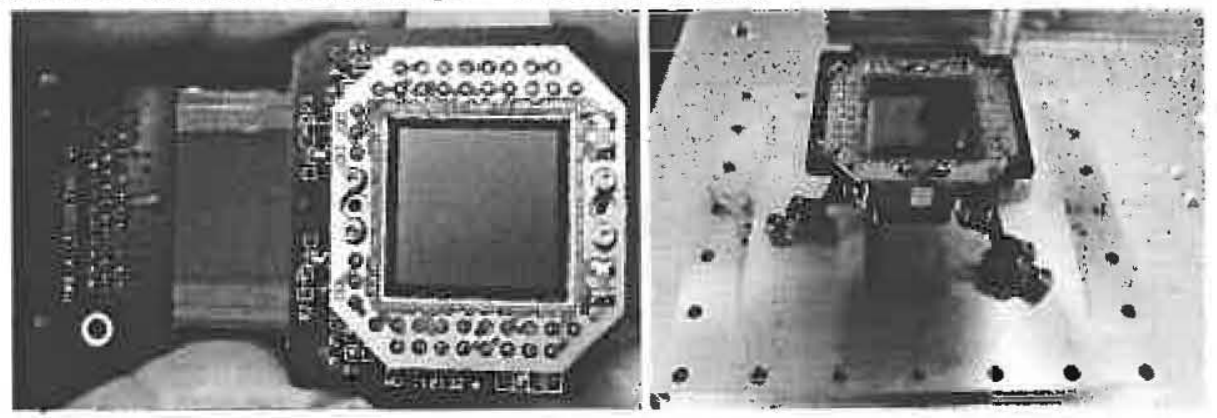

Figure 7. Left: Flex circuit design of detector electronics at focal plane. Right: Top view of detector with flight mount post vibration testing with launch loads.

$3750 \AA$ (dashed lines in Figure 8). The azimuthal and measurement variation was tracked by the witness mirrors located around the perimeter of the primary mirror. Again, the variation is largest in the blue spectral region, with a variation of $0.2-0.3 \%$ at $3750 \AA$ to $4000 \AA$ (solid lines in Figure 8). With the exception of the aluminum absorption feature near $8000 \AA$ the reflectance exceeds $90 \%$ across the ACCESS spectral bandpass (3500 $\AA$ to $1.7 \mu \mathrm{m})$.

\begin{tabular}{|c|c|c|c|c|c|c|}
\hline Mirror & $\begin{array}{c}\text { Al Coating } \\
\text { Thickness }\end{array}$ & $\begin{array}{c}\mathrm{MgF}_{2} \text { Overcoat } \\
\text { Thickness }\end{array}$ & $\begin{array}{l}\text { Reflectivity } \\
\text { at } 3000 \AA\end{array}$ & $\begin{array}{c}\text { Reflectivity } \\
\text { at } 4500 \AA\end{array}$ & $\begin{array}{c}\text { Reflectivity } \\
\text { at } 1.0 \mu \mathrm{m}\end{array}$ & $\begin{array}{c}\text { Reflectivity } \\
\text { at } 1.7 \mu \mathrm{m}\end{array}$ \\
\hline $\begin{array}{l}\text { Primary \& } \\
\text { Secondary }\end{array}$ & $\begin{array}{l}635 \AA \\
635 \AA\end{array}$ & $\begin{array}{l}300 \AA \\
300 \AA\end{array}$ & $89.0 \%$ & $90.3 \%$ & $93.3 \%$ & $96.8 \%$ \\
\hline 18-inch Flat & $640 \AA$ & $297 \AA$ & $89.3 \%$ & $90.4 \%$ & $93.2 \%$ & $96.7 \%$ \\
\hline
\end{tabular}

Table 2. ACCESS Primary and 18-inch Autocollimating flat mirror coating parameters and the average reflectance of the witness mirrors located around the perimeter of the mirror during coating. 

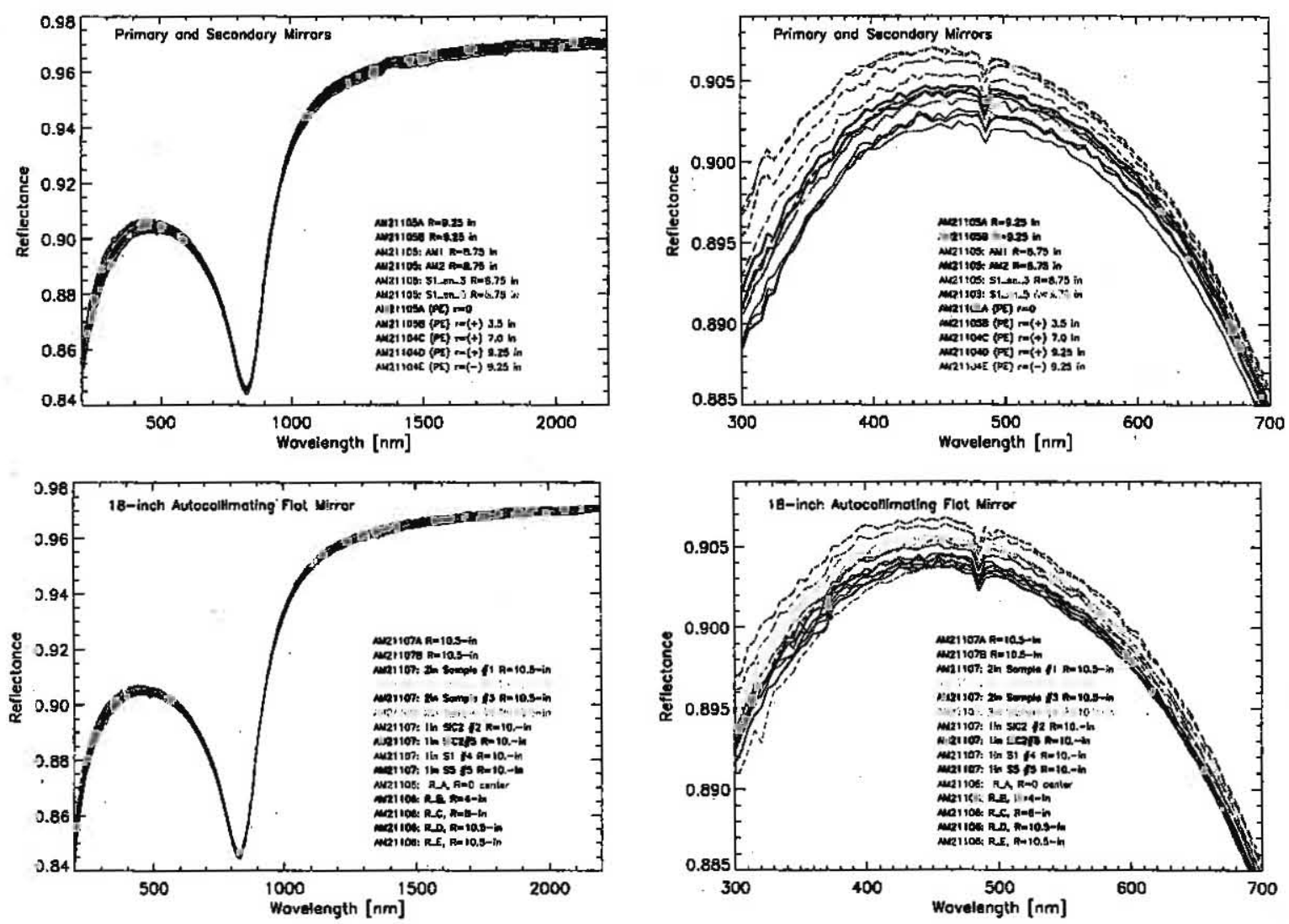

Figure 8. Top Left: Witness mirror reflectance measurements over the full ACCESS bandpass and extending to $2.2 \mu \mathrm{m}$ for the primary and secondary mirror flight coating. The solid lines indicate the reflectivity of the witness samples placed around the perimeter of the mirror during coating. The dashed lines are witness mirror measurements from a test run with the witness mirrors spanning the extent of the primary mirror. These data were used to predicted the radial dependence of the reflectivity due the the radial variation of the thickness of the $\mathrm{MgF}_{2}$ overcoat. For each of the dashed curves, the radial position of the test mirror is presented in the legend. Top Right: Witness mirror reflectance measurements at wavelengths corresponding to the largest reflectance variation in the ACCESS bandpass. Bottom Left: The coating performance for the 18-inch autocollimating flat mirror. The details are as described for the primary mirror (top left). Bottom Right: An expanded view of the reflectivity at the shortest wavelengths in the ACCESS bandpass. The solid lines and dashed lines for all panels are as described for the Top Left panel. 


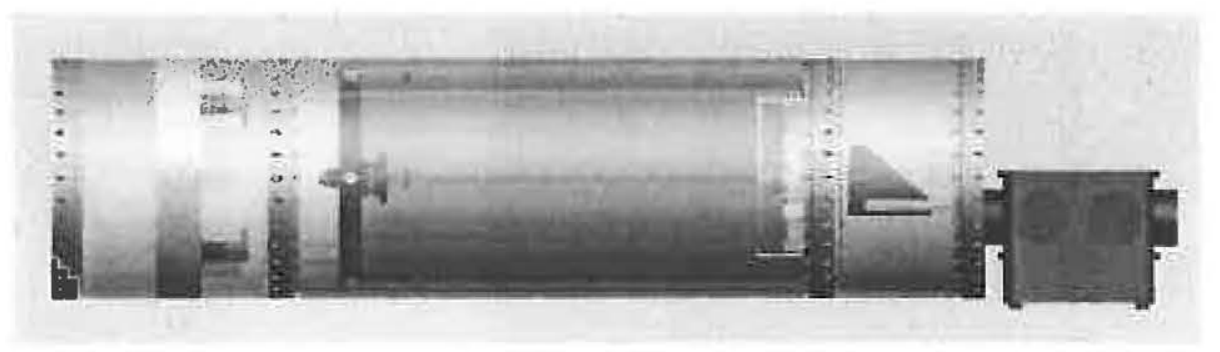

Figure 9. Auto-collimator subsystem used to calibrate the reflectivity of the collimator primary and secondary mirrors. From the right, a light source (not shown) feeds a vacuum monochromator, which is fiber fed into the auto-collimator housing to an order blocking filter and input to an integrating sphere. The output of the integrating sphere is baffled to match the collimator f-ratio. The light then passes through a pinhole at the focus of the collimator and is incident on the NIST standard photodiode detector which is positioned to measure the beam entering or exiting the auto-collimator. The auto-collimating flat mirror (yellow) is on the left. This assembly calibrates the collimator that will be feeding the artificial star-at-infinity to the telescope.

\section{ACCESS CALIBRATION}

\subsection{Calibration Overview}

The crux of the ACCESS instrument sensitivity is knowing the ratio of the total number of photons entering the telescope aperture to the total number of photons detected by the spectrograph detector as a function of wavelength.

An artificial star will be used to provide a known source of photons for the telescope. The artificial star (Fig. 10 Right) consists of an illuminated pinhole placed at the collimator focus. The light illuminating the pinhole originates with a quartz tungsten halogen (QTH) continuum lamp fed to the vacuum monochromator. The output from the monochromator is fiber fed into the collimator vacuum housing. There it illuminates an order blocking filter, followed by an integrating sphere to generate a spatially uniform, unpolarized beam that is input to a baffle box to match the focal ratio of the stellar simulator beam to the F/12 collimator.

The total number of photons in the "star-at-infinity" output beam of the collimator will be provided by two measurements. The first is a measurement of the reflectivity of the auto-collimating flat mirror using a NIST calibrated photodiode detector standard. The second is a measurement of the intensity of the light from the stellar simulator that is input to and reflected by the auto-collimator. Figure 9 illustrates the auto-collimator calibration configuration used to determine the reflectivity of the primary and secondary mirrors. The absolute calibration transfer is obtained through the use of a NIST calibrated photodiode detector transfer standard to measure the input and output images at the auto-collimator focal plane.

The end-to-end calibration of the telescope with spectrograph (Fig. 10: Right) is then performed as a function of wavelength by measuring the intensity of the simulated star, measuring the count rate at the spectrograph detector, correcting for the collimator attenuation of the simulated star by the reflectivity of the collimator primary and secondary mirrors, and dividing the calibrated radiant flux by the illuminated area of the primary mirror.

Systematic effects, such as the uniformity of reflective coatings, matching of the collimator and telescope apertures, the spatial uniformity of the photodiode detectors, the transmission of the slit, the scattered light determination, the determination of the area of the primary and secondary telescope mirrors, the stability of the light source, etc., must be closely tracked if this process is to yield the required precision and accuracy.

In addition to the calibration of the ACCESS payload to a fundamental irradiance standard, ACCESS will be calibrated to both continuum and emission line fundamental radiance standards. Using emission from tunable lasers, the NIST SIRCUS facility ${ }^{15}$ will provide an end-to-end radiance calibration transfer to ACCESS while the use of a spectral light engine will calibrate ACCESS using a continuum spectral energy distribution similar to the spectral energy distribution of the stellar targets. ${ }^{16}$ 

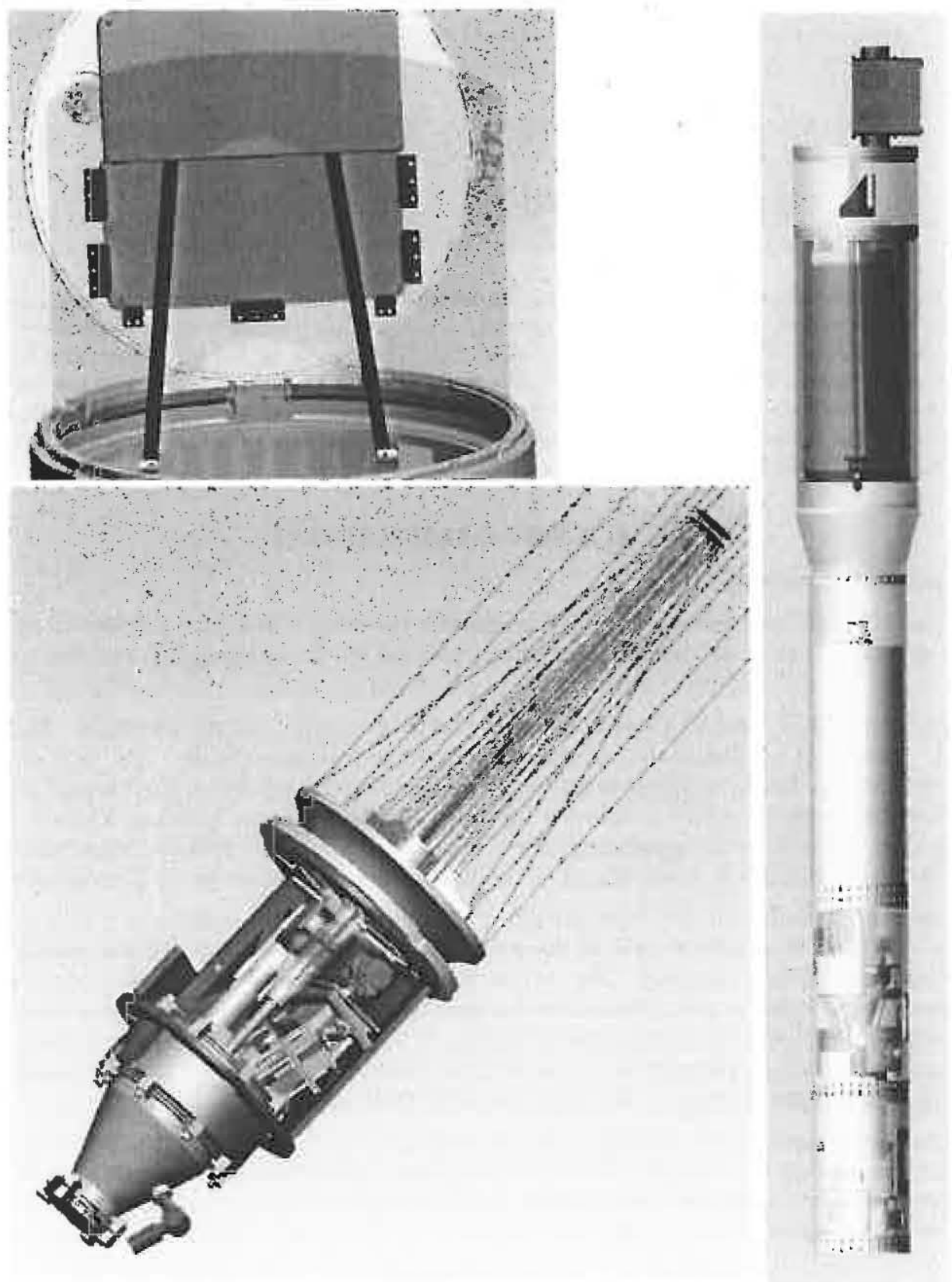

Figure 10. Top Left: The telescope aperture door in the open position with the spectralon diffuser is mounted on the inside. To maintain an unvignetted beam from the target star, the lower half of the diffuser is mounted under the arms that extend to open the aperture door during flight. Bottom Left: The ACCESS telescope and spectrograph (not all components are shown). The inner evacuated spectrograh housing is shown in green, the detector housing, cooler and radiation shiels are on the right. A subset of the optical bench (purple) is shown to enable a view of the slit, detector subsystem and guide camera. Right: Assembled ground calibration configuration of ACCESS with the collimated artificial star. From the top, a light source (not shown) feeds a monochromator, which is fiber fed to an order blocking filter and input to an integrating sphere. The output of the integrating sphere is baffled to match the collimator f-ratio. The light then passes through a pinhole at the focus of the collimator. This resulting collimated beam is the calibrated light source for the ACCESS instrument. 


\subsection{Calibration Monitoring}

An On-board Calibration Monitor (OCM) will track sensitivity changes in the telescope and spectrograph as a function of time before and after the absolute calibration data have been collected. ${ }^{7,8,17}$ The OCM $^{17}$ uses 8 pairs of feedback stabilized LEDs, with central wavelengths spanning the ACCESS bandpass, to illuminate the telescope by scattering off a diffuser mounted on the interior of the telescope cover (Fig. 10). The LEDs are located in a multi-layer annular assembly mounted as a collar around the nose of the star tracker positioned behind the secondary mirror of the telescope. This assembly does not increase the central obscuration of the cassegrain telescope. Feedback stabilization is achieved through brightness monitoring of each LED by an adjacent dedicated photodiode. The OCM will monitor instrument performance during the end-to-end transfer of the NIST calibration of the diode standards to ACCESS in the laboratory. This light source will provide the capability to switch on-off during an observation to check the detector dependence on count rate.

\section{STATUS}

The ACCESS instrument and calibration hardware are in an active fabrication phase. ${ }^{18}$ The telescope and collimator mirrors have been coated. The housing for the collimator and auto-collimating flat mirror systems have been fabricated. The collimator optics are ready for integration. The 18-inch flat mirror mount, rotary and translation stages have been fabricated, assembled, and control software written and tested. The flight detector has been selected and a subset of performance tests have been presented here. The detector mount assembly (Fig. 7) and housing have been vibration tested at launch levels and thermal acceptance testing has been performed at $130 \mathrm{~K}$. The first launch is expected within the year.

\section{SUMMARY}

ACCESS is an active sub-orbital program with a payload development component followed by four launches over the subsequent two years. This experiment will enable a fundamental calibration of the spectral energy distribution of established bright primary standard stars, as well as standard stars 10 magnitudes fainter, in physical units though a direct comparison with NIST traceable irradiance (detector) standards. Each star will be observed on two separate rocket flights to verify repeatability to $<1 \%$, an essential element in establishing standards with $1 \%$ precision.

\section{ACKNOWLEDGMENTS}

This research is being funded through NASA APRA-2007.

\section{REFERENCES}

[1] Hayes, D. S. and Latham, D. W., "A rediscussion of the atmospheric extinction and the absolute spectralenergy distribution of VEGA," Ap. J. 197, 593-601 (May 1975).

[2] Hayes, D. S., "Stellar absolute fluxes and energy distributions from 0.32 to 4.0 microns," in [IAU Symp. 111: Calibration of Fundamental Stellar Quantities], 225-249, Reidel, Dordrecht (1985).

[3] Oke, J. B. and Schild, R. E., "The Absolute Spectral Energy Distribution of Alpha Lyrae," Ap. J. 161, 1015 (Sept. 1970).

[4] Hill, G., Gulliver, A. F., and Adelman, S. J., "A Study of Vega: A Rapidly Rotating Pole-On Star," 712, 250-259 (Mar. 2010).

[5] Yoon, J., Peterson, D. M., Zagarello, R. J., Armstrong, J. T., and Pauls, T., "The Effect of Rotation on the Spectrum of Vega," 681, 570-578 (July 2008).

[6] Aufdenberg, J. P., Mérand, A., Coudé du Foresto, V., Absil, O., Di Folco, E., Kervella, P., Ridgway, S. T., Berger, D. H., ten Brummelaar, T. A., McAlister, H. A., Sturmann, J., Sturmann, L., and Turner, N. H., "First Results from the CHARA Array. VII. Long-Baseline Interferometric Measurements of Vega Consistent with a Pole-On, Rapidly Rotating Star," 645, 664-675 (July 2006). 
[7] Kaiser, M. E., Kruk, J. W., McCandliss, S. R., Sahnow, D. J., Barkhouser, R. H., Van Dixon, W., Feldman, P. D., Moos, H. W., Orndorff, J., Pelton, R., Riess, A. G., Rauscher, B. J., Kimble, R. A., Benford, D. J., Gardner, J. P., Hill, R. J., Woodgate, B. E., Bohlin, R. C., Deustua, S. E., Kurucz, R., Lampton, M., Perlmutter, S., and Wright, E. L., "ACCESS: Enabling an Improved Flux Scale for Astrophysics," $\mathrm{ArXiv}$ e-prints http://adsabs.harvard.edu/abs/2010arXiv1001.3925K (Jan. 2010).

[8] Kaiser, M. E., Kruk, J. W., McCandliss, S. R., Sahnow, D. J., Rauscher, B. J., Benford, D. J., Bohlin, R. C., Deustua, S. E., Dixon, W. V., Feldman, P. D., Gardner, J. P., Kimble, R. A., Kurucz, R., Lampton, M., Moos, H. W., Perlmutter, S., Riess, A. G., Woodgate, B. E., and Wright, E. L., "ACCESS: absolute color calibration experiment for standard stars," in [Society of Photo-Optical Instrumentation Engineers (SPIE) Conference Series], 7014 (Aug. 2008).

[9] Fukugita, M., Ichikawa, T., Gunn, J. E., Doi, M., Shimasaku, K., and Schneider, D. P., "The Sloan Digital Sky Survey Photometric System," Ap. J. 111, 1748 (F96) (Apr. 1996).

[10] Reach, W. T., Megeath, S. T., Cohen, M., Hora, J., Carey, S., Surace, J., Willner, S. P., Barmby, P., Wilson, G., Glaccum, W., Lowrance, P., Marengo, M., and Fazio, G. G., "Absolute Calibration of the Infrared Array Camera on the Spitzer Space Telescope," PASP 117, 978-990 (Sept. 2005).

[11] Moreels, G., Megie, G., Vallance Jones, A., and Gattinger, R. L., "An oxygen-hydrogen atmospheric model and its application for the $\mathrm{OH}$ emission problem.," Journal of Atmospheric and Terrestrial Physics 39, 551-570 (1977).

[12] Féry, C., "A Prism with Curved Faces, for Spectrograph or Spectroscope," 34, 79- (July 1911).

[13] McCandliss, S. R., Martinez, M. E., Feldman, P. D., Pelton, R., Keski-Kuha, R. A., and Gum, J. S., "Design and fabrication of a 40-cm-diameter SiC-coated normal incidence telescope and spectrometer," in [Society of Photo-Optical Instrumentation Engineers (SPIE) Conference Series], 2011, 310 (1994).

[14] Hill, R. J., Malumuth, E. M., Foltz, R. D., Kimble, R. A., Waczynski, A., Boehm, N., Wen, Y., Kan, E., and Collins, N. R., "Reciprocity failure in 1.7 micron cut-off hgcdte detectors," in [Society of Photo-Optical Instrumentation Engineers (SPIE) Conference Series], 7731, 115 (2010).

[15] Brown, S. W., Eppeldauer, G. P., and Lykke, K. R., "Facility for spectral irradiance and radiance responsivity calibrations using uniform sources," 45, 8218-8237 (Nov. 2006).

[16] Brown, S. W., Rice,'J. P., Neira, J. E., C., J. B., and Jackson, J. D., "'Spectrally Tunable Sources for Advanced Radiometric Applications."," 111, 401-410 (Nov. 2006).

[17] Kruk, J. W., Kaiser, M. E., McCandliss, S. R., Orndorf, J., Barkhouser, R. H., Sahnow, D. J., Benford, D. J., Bohlin, R. C., Deustua, S. E., Dixon, W. V., Feldman, P. D., Gardner, J. P., Kimble, R. A., Kurucz, R., Lampton, M., Moos, H. W., Perlmutter, S., Rauscher, B. J., Riess, A. G., Woodgate, B. E., and Wright, E. L., "On-board calibration monitor for tracking instrument sensitivity," in [Society of Photo-Optical Instrumentation Engineers (SPIE) Conference Series], 7014, 115 (Aug. 2008).

[13] Kaiser, M. E., Kruk, J. W., McCandliss, S. R., Rauscher, B. J., Kimble, R. A., Pelton, R. S., Sahnow, D. J., Dixon, W. V., Feldman, P. D., Gaither, B. W., Lazear, J. S., Moos, H. W., Riess, A. G., Benford, D. J., Gardner, J. P., Hill, R. J., Kahle, D. M., Mott, D. B., Waczynski, A., Wen, Y., Woodgate, B. E., Bohlin, R. C., Deustua, S. E., Kurucz, R., Lampton, M., Perlmutter, S., and Wright, E. L., "ACCESS: design and preliminary performance," in [Society of Photo-Optical Instrumentation Engineers (SPIE) Conference Series], 7731, 112 (July 2010). 